\title{
Surgical Treatment of Ankylosing Spondylitis with Andersson Lesion
}

\author{
Fengguang Yang, Liang Yang, Enhui Ren, Yong Yang, Jing Wang and Xuewen Kang \\ Department of Orthopedics, Lanzhou University Second Hospital, Lanzhou, PR China
}

\begin{abstract}
Ankylosing spondylitis (AS) with an Andersson lesion may cause kyphosis. As the disease progresses, kyphosis gradually worsens. When conservative treatment does not improve the symptoms, surgery is often needed to correct the deformity. The optimal surgical approach for ankylosing spondylitis with kyphosis remains controversial. Here, we introduce our surgical procedure of pseudoarticular debridgement and osteotomy of the apical vertebrae of the Cobb angle. We found that both the kyphosis and associated symptoms improved significantly after 13 months of follow-up.
\end{abstract}

Key Words: Ankylosing spondylitis, Andersson lesion, Kyphosis.

How to cite this article: Yang F, Yang L, Ren E, Yang Y, Wang J, Kang X. Surgical treatment of ankylosing spondylitis with Andersson lesion. J Coll Physicians Surg Pak 2019; 29 (Supplement 2):S135-S137.

\section{INTRODUCTION}

Andersson lesion $(\mathrm{AL})$ destroys the vertebral body or intervertebral disc and develops into ankylosing spondylitis (AS), in the late stages, usually because of minor trauma. The lesion may cause back pain, neurological deficits, and progressive kyphosis, rendering treatment essential. ${ }^{1}$ The goals of surgery are vertebral fusion, reduction of the neurological deficits, and restoration of a normal spine. ${ }^{2}$ However, the optimal surgical approach remains controversial. ${ }^{3}$ Several procedures have been proposed, including posterior and anterior fusion, and a combined approach. ${ }^{4}$ However, the outcomes have not been ideal and different patients may require different procedures. Here, we present an example of successful treatment.

\section{CASE REPORT}

A male, aged 42 years, was admitted to our hospital in October 2017 with history of 12 years of increasing back pain and one month of unstable walking. We obtained written informed consent to use his personal information. The patient remarked that his lumbar back stiffness, which started 12 years ago, had no obvious cause. In hospital, he was initially diagnosed with rheumatoid arthritis, followed by AS. Three years ago, he had experienced several falls and, nine months prior to admission to our hospital, he developed lower back pain and kyphosis (Figure 1A), and could not walk stably.

Correspondence to: Xuewen Kang, Department of Orthopedics,

Lanzhou University Second Hospital, 82 Cuiying Men,

Lanzhou 730000, Gansu Province, PR China

E-mail:ery_kangxw@lzu.edu.cn

Received: March 01, 2019; Revised:May 18, 2019;

Accepted: May 29, 2019
Physical examination revealed a C-shaped kyphotic deformity, a chin-brow vertical angle (CBVA) of $51^{\circ}$, limited left hip joint flexion, extension, abduction, adduction, rotation, increased muscle tension in both lower limbs, grade $\mathrm{V}$ muscle strength of both lower limbs, and lower limb skin tautness. The normal physiological reflexes were present and no pathology was noted. The visual analog scale (VAS) pain score was 7 and the Oswestry dysfunction index (ODI) score was $71.3 \%$. X-ray radiography and computed tomography (CT) revealed a bamboo-like change across the entire spine, a pseudo-articular formation between T11/T12, and obvious hyperplasia (Figure 2A). Magnetic resonance imaging (MRI) revealed thick ossification of the ligamentum flavum in the spinal canal of T11/T12, in addition to spinal stenosis (Figure $2 \mathrm{~B}$ ). The erythrocyte sedimentation rate was $5 \mathrm{~mm} / \mathrm{h}$ and his HLA-B27 status was negative. Under general anesthesia, we performed joint debridement and osteotomy. After placing the patient in the prone position, the abdomen was padded, a waist bridge was placed, and a posterior median incision was made to expose the lamina. The surgical region ran from T10 to L4; the length of the T10-L1 and L3-L4 vertebral bodies was 40 and $45 \mathrm{~mm}$, respectively. We placed pedicle screws, removed the T11-T12 lamina, thickened ossified (yellow) ligament, and dura mater, protected the spinal cord, and revealed the T11-T12 intervertebral space (and noted any destruction thereof). Purulent tissue was found to be absent. We, then scraped the fibrous tissue of the intervertebral space and hardened the bone, implanted fragmented bone into the intervertebral space (near the top vertebrae of the Cobb angle), exposed and broke both articular joints, performed a wedge-shaped osteotomy via the L2 pedicles, placed pre-bent connecting rods after treating a hemorrhage, and pressurised the thoracolumbar 


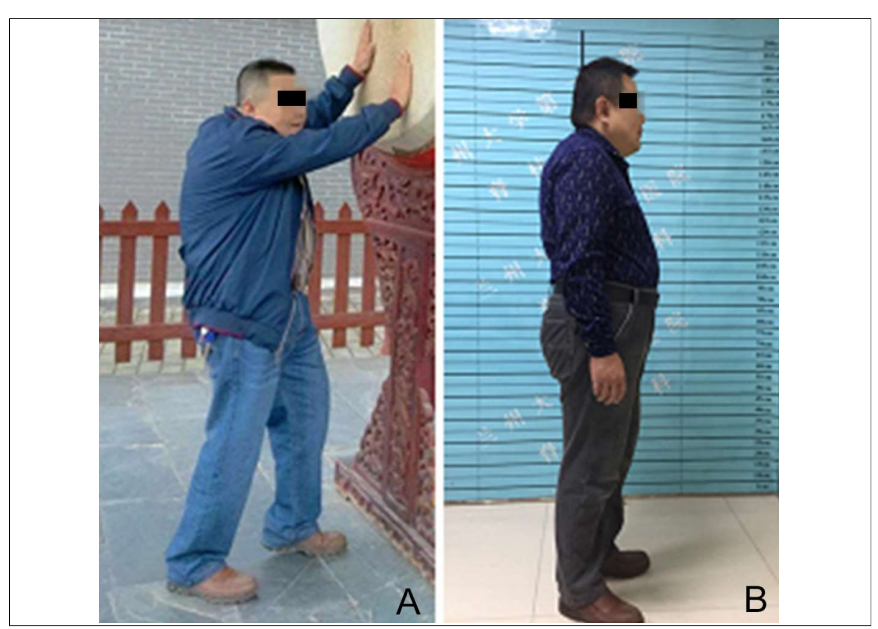

Figure 1: (A) Preoperative. (B) Postoperative.

segments. The kyphosis was oriented to the desired position (the CBVA was approximately $0^{\circ}$ ) and the nut was tightened. After waking, the patient exhibited good lower limb function.

The intraoperative blood loss was $620 \mathrm{~mL}$; three units of red blood cells and $350 \mathrm{~mL}$ of plasma were infused. At the 6-month follow-up, the patient could lie supine while breathing smoothly, and his walking posture had significantly improved. The Cobb angle was $10^{\circ}$ and the CBVA was $0^{\circ}$ (Figure 1B). The VAS pain score was 1 and the ODI score was 19.2. Bone fusion of the pseudoarticular joint was observed on thoracolumbar CT (Figure 2C) and X-ray (Figure 2D). In the postoperative full-spine side view (Figure 2E), the spinal curvature was near-physiological. The kyphosis and associated symptoms showed significant improvement at the 13-month follow-up.

\section{DISCUSSION}

AS is a chronic inflammatory disease characterised by erosion of the spine and ankle joints. ${ }^{5}$ As the disease progresses, late calcification and ossification of soft tissue around the entire spine triggers kyphosis. ${ }^{6}$ In patients with AS, the greatest shear/stress is located in the thoracolumbar region, which is prone to extensive fracture due to ossification. ${ }^{7}$ Surgery is essential, if conservative treatment proves ineffective. 4,8

Recently, a single back-end approach to AL has become increasingly popular. Liang et al. performed posterior wedge osteotomy to treat $A L$ in a patient with kyphosis and AS, 9 The follow-up ODI and VAS scores, as well as the fusion outcome, were satisfactory; but there was no discussion of any improvement in the spinal deformity. Zhang et al. performed osteotomy via transpedicular decompression and discectomy, and reported satisfactory results. ${ }^{8}$ Wang et al. used a single posterior approach to remove the pseudo-arthrosis and placed bone grafts; fusion strength was good, but deformity correction was not discussed. 10

After 3 years of rest, and prescription of non-steroidal anti-inflammatory drugs (NSAIDs) and sulfa drugs, the symptoms of our patient had not improved and the kyphosis deformity had worsened, seriously affecting his quality of life. Therefore, we performed pseudo-articular debridement and osteotomy of the apical vertebrae of the Cobb angle. Prior to surgery, we found that the T11T12 region exhibited stenosis and pseudo-articular joints, and that the apical vertebrae of the Cobb angle were located in the L2 vertebral body. During surgery, we performed total laminectomy and decompression of the stenotic region, to relieve the spinal cord compression and preserve the removed bone under reduced pressure. After the proliferative hard tissue was removed, the decompressed bone was implanted into the T11-T12 gap to promote healing. We chose the $L 2$ pedicle as the site for subsequent wedge-shaped osteotomy, because this was the most obvious component of the deformity. We believed that osteotomy would be optimal for correction of the kyphosis. Finally, we inserted a spinal rod to maintain the CBVA close to $0^{\circ}$. On follow-up, the symptoms were greatly reduced and the spinal deformity was well-restored.

In general, pseudo-articular debridement and osteotomy of the apical vertebrae of the Cobb angle maximise the correction of kyphosis, and render the spine more
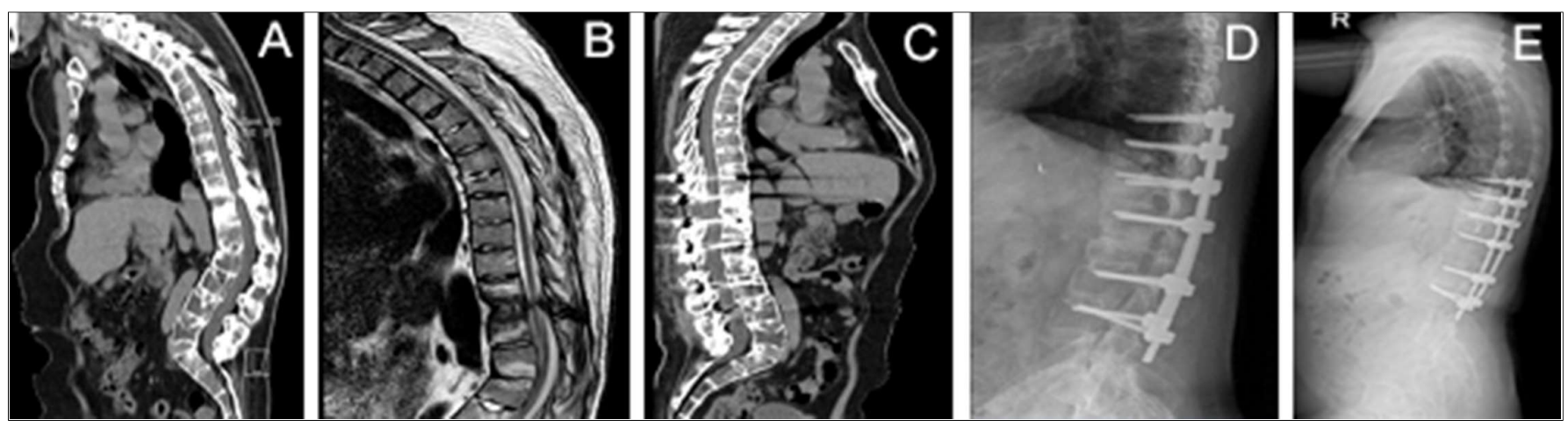

Figure 2: Preoperative and postoperative imaging changes. (A) Preoperative sagittal CT shows the formation of thoracolumbar pseudo-arthrosis, thoracolumbar kyphosis. (B) Preoperative MRI shows T11-T12 thickening of the ligamentum flavum in the spinal canal, spinal stenosis, spinal cord compression. (C) and (D) CT and X-rays at 6 months after operation showed bony fusion at the pseudo-arthrosis and osteotomy sites, and the thoracic and lumbar kyphosis became significantly better. (E) Spine curvature close to physiological curvature on X-ray film after 6 months of operation. 
balanced in the sagittal position. This approach also restores normal stature and relieves clinical symptoms

\section{PATIENT'S CONSENT:}

Written consent was taken from the patient.

\section{CONFLICT OF INTEREST:}

Authors declared no conflict of interest.

\section{AUTHORS' CONTRIBUTION:}

$F Y, Y Y, X K$ : Orthopaedic surgery.

LY, ER, JW: Data collection and follow-up.

FY, XK: Literature search and final draft of the case report.

\section{REFERENCES}

1. Kim KT, Lee SH, Suk KS, Lee JH, Im YJ. Spinal pseudarthrosis in advanced ankylosing spondylitis with sagittal plane deformity: clinical characteristics and outcome analysis. Spine (Phila Pa 1976) 2007; 32:1641-7.

2. Bron JL, de Vries MK, Snieders MN, van Der Horst-Bruinsma IE, van Royen BJ. Discovertebral (Andersson) lesions of the spine in ankylosing spondylitisrevisited. Clin Rheumatol 2009; 28 : 883-92.

3. Dave BR, Ram H, Krishnan A. Andersson lesion: Are we misdiagnosing it? A retrospective study of clinico-radiological features and outcome of short segment fixation. Eur Spine $J$ 2011; 20:1503-9.

4. Chang KW, Tu MY, Huang HH, Chen HC, Chen YY, Lin CC. Posterior correction and fixation without anterior fusion for pseudoarthrosis with kyphotic deformity in ankylosing spondylitis. Spine (Phila Pa 1976) 2006; 31:E408-13.

5. Feldtkeller E, Vosse D, Geusens $P$, van der Linden S. Prevalence and annual incidence of vertebral fractures in patients with ankylosing spondylitis. Rheumatol Int 2006; 26:234-9.

6. Peh WC, Luk KD. Pseudoarthrosis in ankylosing spondylitis. Ann Rheum Dis 1980; 53:206-10.

7. Van Royen BJ, Kastelijns RC, Noske DP, Oner FC, Smit TH. Transpedicular wedge resection osteotomy for the treatment of a kyphotic Andersson lesioncomplicating ankylosing spondylitis. Eur Spine J 2006; 15:246-52.

8. Zhang X, Wang Y, Wu B, Hu W, Zhang Z, Wang Y. Treatment of Andersson lesion-complicating ankylosing spondylitis via transpedicular subtraction and disc resection osteotomy, a retrospective study. Eur Spine $J$ 2016; 25:2587-95.

9. Liang $Y$, Tang $X$, Zhao $Y$, Wang Z. Posterior wedge osteotomy and debridement for Andersson lesion with severe kyphosis in ankylosing spondylitis. J Orthop Surg Res 2017; $12: 54$.

10. Wang T, Wang D, Cong Y, Yin C, Li S, Chen X. Evaluating a posterior approach for surgical treatment of thoracolumbar pseudarthrosis in ankylosing spondylitis. Clin Spine Surg 2017; 30:E13-8. 